\title{
Retinal microvascular calibre and risk of diabetes mellitus: a systematic review and participant-level meta-analysis
}

\author{
Charumathi Sabanayagam ${ }^{1,2,3}$ • Weng Kit Lye ${ }^{4}$. Ronald Klein ${ }^{5}$ - Barbara E. K. Klein ${ }^{5}$. \\ Mary Frances Cotch ${ }^{6}$ • Jie Jin Wang ${ }^{7}$ - Paul Mitchell ${ }^{7}$ - Jonathan E. Shaw ${ }^{8}$. \\ Elizabeth Selvin $^{9}$ • A. Richey Sharrett ${ }^{9}$ - Tien Y. Wong ${ }^{1,2,3}$
}

Received: 30 April 2015 / Accepted: 8 July 2015 / Published online: 2 August 2015

(C) Springer-Verlag Berlin Heidelberg 2015

\begin{abstract}
Aims/hypothesis The calibre of the retinal vessels has been linked to diabetes mellitus but studies have not shown consistent results. We conducted a participant-level meta-analysis to evaluate the association between retinal arteriolar and venular calibre and diabetes.

Methods We performed a systematic review on MEDLINE and EMBASE for articles published up to December 2014. We identified five population-based prospective cohort studies that provided individual-level data on 18,771 diabetes-free participants. We used discrete time proportional hazards models to estimate pooled HRs of diabetes associated with 1 SD $(20 \mu \mathrm{m})$ change in retinal vascular calibre.

Results We identified 2,581 incident cases of diabetes over a median follow-up period of 10 years (interquartile interval of 3.4-15.8 years). After adjustment for demographic, lifestyle and clinical factors, retinal venular calibre was significantly associated with incident diabetes (pooled HR 1.09 [95\% CI $1.02,1.15]$ per SD increase in venular calibre). This association persisted in analyses excluding individuals with $<5$ years of follow-up $(1.07[1.0,1.12])$ or those with impaired fasting
\end{abstract}

Tien Y. Wong

ophwty@nus.edu.sg

1 Singapore Eye Research Institute, Singapore National Eye Centre, 11 Third Hospital Avenue, Singapore 168751, Republic of Singapore

2 Ophthalmology and Visual Sciences Academic Clinical Program, Duke-NUS Graduate Medical School, Singapore, Republic of Singapore

3 Department of Ophthalmology, National University of Singapore, Singapore, Republic of Singapore

4 Centre for Quantitative Medicine, Duke-NUS Graduate Medical School, Singapore, Republic of Singapore glucose at baseline $(1.10[1.03,1.17])$; in subgroup analyses, the association was stronger in men than in women but was consistent across subgroups of race/ethnicity, smoking status, hypertension and BMI categories. Retinal arteriolar calibre was not associated with diabetes $(0.95[0.86,1.06]$ per SD decrease in arteriolar calibre).

Conclusions/interpretation Wider retinal venules but not narrower retinal arterioles were associated with a modestly increased risk for diabetes. Knowledge of pathological mechanisms underlying wider retinal venule may provide further insights concerning microvascular alterations in diabetes.

Keywords Cohortstudies $\cdot$ Diabetes $\cdot$ Meta-analysis $\cdot$ Retinal vessels $\cdot$ Systematic review

\section{Abbreviations \\ ARIC Atherosclerosis Risk in Communities study \\ AusDiab Australian Diabetes, Obesity and Lifestyle study AVR Arteriole-to-venule ratio}

5 Department of Ophthalmology and Visual Sciences, School of Medicine and Public Health, University of Wisconsin, Madison, WI, USA

6 Division of Epidemiology and Clinical Applications, National Eye Institute, National Institutes of Health, Bethesda, MD, USA

7 Department of Ophthalmology and Westmead Millennium Institute, Centre for Vision Research, University of Sydney, Sydney, NSW, Australia

8 Baker IDI Heart and Diabetes Institute, Melbourne, VIC, Australia

9 Department of Epidemiology, John Hopkins Bloomberg School of Public Health, Baltimore, MD, USA 


$\begin{array}{ll}\text { BDES } & \text { Beaver Dam Eye Study } \\ \text { BMES } & \text { Blue Mountains Eye Study } \\ \text { CRAE } & \text { Central retinal arteriolar equivalent } \\ \text { CRVE } & \text { Central retinal venular retinal equivalent } \\ \text { CVD } & \text { Cardiovascular disease } \\ \text { IVAN } & \text { Interactive Vessel Analysis } \\ \text { MESA } & \text { Multi-Ethnic Study of Atherosclerosis }\end{array}$

\section{Introduction}

Diabetes mellitus is a major public health problem estimated to affect 371 million people worldwide [1]. In 2010, diabetes was estimated to affect 26 million adults in the USA alone [2]. The pathogenesis of diabetes is multifactorial and still not fully established. Microvascular dysfunction has been postulated to play an important role in the pathogenesis of diabetes $[3,4]$. Microvascular abnormalities such as arteriolar narrowing and impaired microvascular perfusion delay the access of glucose and insulin to target tissues, which may lead to insulin resistance [5], a major mechanism underlying type 2 diabetes. In experimental studies, correlation of both insulininduced capillary recruitment in skin [4] and insulin-mediated microvascular recruitment in muscle [6] provide evidence to support the role of small vessel disease in insulin resistance.

The retinal microvasculature represented by retinal arterioles and venules (100-300 $\mu \mathrm{m}$ in size) offers a unique opportunity for noninvasive visualisation of the systemic microvasculature [7, 8]. Imaging software methods have provided a means to measure subtle abnormalities in the retinal microvasculature such as the calibre of the retinal vessels [9]. The calibre of the retinal microvasculature could reflect impaired microvascular function and microvascular perfusion. In addition, retinal microvascular changes could also represent other shared mechanisms underlying the pathogenesis of diabetes [10], including oxidative stress, endothelial dysfunction, inflammation and hypertension [11-14].

Several cross-sectional studies have shown an association between retinal microvascular calibre and diabetes [15-17]. However, evidence from prospective studies is mixed [18]. While three studies reported smaller retinal arterioles to be associated with diabetes [19-21], one reported that both wider retinal arterioles and venules [17] were associated with diabetes and two studies reported no association between retinal microvascular calibre and diabetes [22, 23]. A recent metaanalysis [24] summarised evidence from published aggregate data of prospective studies on the role of microvascular dysfunction assessed using several biomarkers including retinal microvascular variables. This meta-analysis reported smaller retinal arteriole-to-venule ratio (AVR) to be associated with incident diabetes based on published data from three studies $[19,20,23]$. However, in analyses including retinal arteriolar and venular calibre separately, neither one showed a significant association with incident diabetes [24]. To clarify the association between retinal vascular calibre and diabetes, we conducted a systematic review and an individual participant-level meta-analysis of prospective cohort studies to estimate the risk of diabetes associated with retinal microvascular calibre. We hypothesised that narrower retinal arterioles and wider retinal venules would be associated with an increased risk of diabetes.

\section{Methods}

Data extraction We (C. Sabanayagam and T. Y. Wong) performed a systematic search of the MEDLINE (PubMed), and EMBASE databases up to December 2014. Conference proceedings and reference lists of selected articles were also manually scanned to identify possible additional studies. The following terms were used for the MEDLINE search: (exp retinal diseases/, retinopathy.tw., (retina or retinal).tw., microvessel.mp. or microvascular.tw., vessel.mp. or vascular.tw., arteriole.mp. or arteriolar.tw., venule.mp. or venular.tw.) and (diabetes.mp. or Diabetes Mellitus, Type 2/ or Diabetes Mellitus/) and (exp epidemiology/, exp epidemiologic studies/, incidence/, exp prognosis/, predict\$.mp., prognos\$.tw., risk.tw.). Similar search terms were used for EMBASE. We restricted our search to English language and human studies. Studies were included if they were carried out in general populations, included measurement of retinal vascular calibre and had documented diabetes incidence. Studies were excluded if they were cross-sectional or if relevant data were unavailable. We conducted our systematic review as proposed by the Meta-analysis of Observational Studies in Epidemiology (MOOSE) guidelines [25].

Data collection We invited the principal investigators of the selected studies to participate in this collaborative project who upon acceptance were requested to provide individual participant data on retinal vascular calibre measurements, incident diabetes and baseline measurements of traditional risk factors included in the Framingham Risk Score [26], such as age, sex, race/ethnicity, current smoking and drinking status, height, weight, systolic and diastolic BP, blood glucose, serum total cholesterol, history of cardiovascular disease (CVD), diabetes and hypertension, and use of glucose-lowering and antihypertensive medication. Since family history was available only in a subset of participants and measures of physical activity were highly variable across the studies, these variables were not considered for inclusion in the analyses. For the present analysis, we excluded individuals with prevalent diabetes or preexisting CVD or those with missing data on covariates included in the multivariable model. We included those who had values for retinal vascular calibres and information on incident diabetes and covariates. 
Studies included We identified 1,400 studies with our initial search strategy (Fig. 1). We then identified 16 studies that had assessed retinal calibres and diabetes in a general population. After screening these studies based on inclusion and exclusion criteria, ten studies were excluded due to the cross-sectional study design or multiple publications from the same study samples, leaving six studies eligible for the meta-analysis. One study (Rotterdam Study) [12] did not have data available at the time of analysis; therefore, five studies were included in the current meta-analysis: the Atherosclerosis Risk in Communities (ARIC, USA) study [19], the Australian Diabetes, Obesity and Lifestyle (AusDiab, Australia) study [21], the Beaver Dam Eye Study (BDES, USA) [20], the Blue Mountains Eye Study (BMES, Australia) [22] and the Multi-ethnic Study of Atherosclerosis (MESA, USA) [17]. Investigators from the five studies agreed to provide data for the individual participant-level meta-analysis.

Measurement of retinal vascular calibre Measurement of retinal vessel calibre was similar in each study, with slight variations. Briefly, for participants of each study, either digital or $35 \mathrm{~mm}$ retinal photographs centred on the optic disc and macula for a single or both eyes were taken at baseline. Stereoscopic $30^{\circ}$ retinal photographs were taken using a

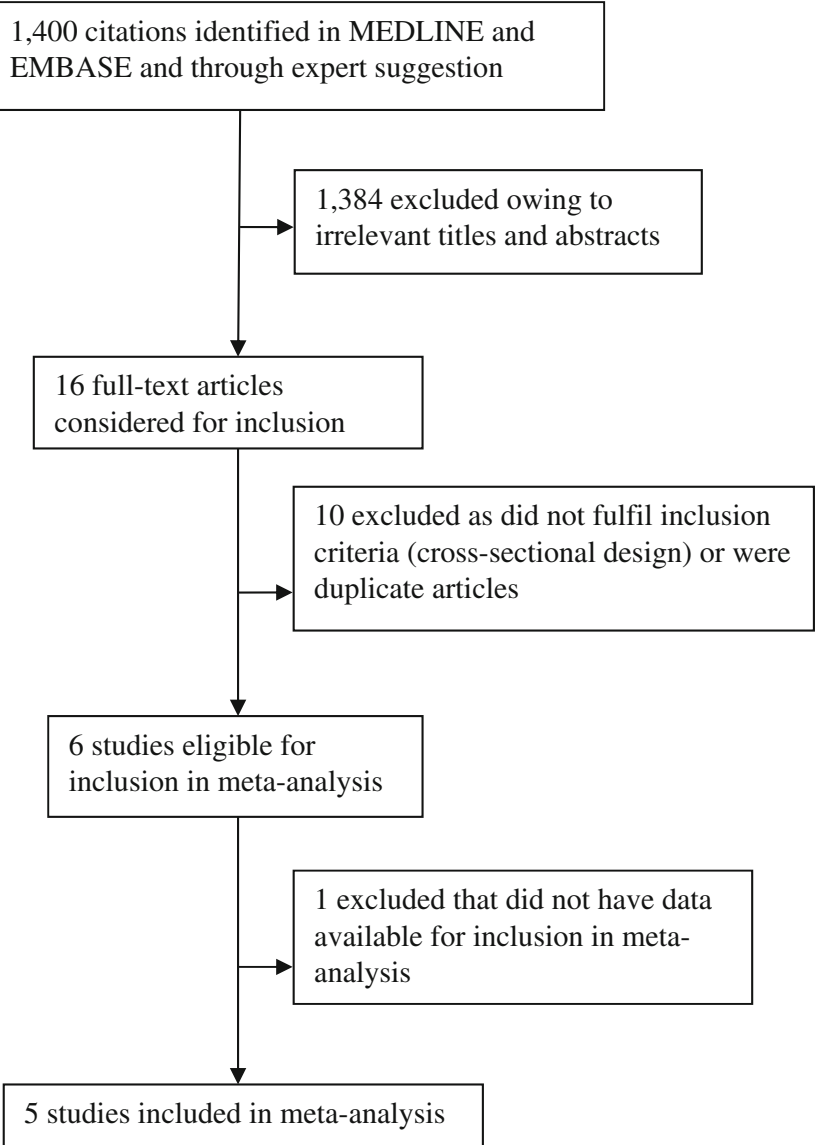

Fig. 1 Search strategy for studies included in the meta-analysis
Zeiss FF3 camera (Carl Zeiss Meditech, Jena, Germany) after pupil dilation in BDES and BMES; $45^{\circ}$ nonmydriatic retinal photographs were taken using a Canon CR6-45NM camera (Canon, Tokyo, Japan) in ARIC and MESA; and a Canon CR45UAF camera and $45^{\circ}$ fields were used in AusDiab. In all studies except ARIC, a pre-specified eye, usually the right eye, was used for the measurement and analysis of retinal vascular calibre. Measurements from the left eye were used when measurements from the right eye could not be used. In ARIC, retinal photographs were taken of one randomly selected eye.

Photographs from the three US studies were graded for retinal vascular measurements at the Ocular Epidemiology Reading Center at the University of Wisconsin, Madison using a computer-assisted software program (Interactive Vessel Analysis [IVAN], University of Wisconsin, Madison, WI, USA) that measured the six largest arterioles and venules. Photographs from the two Australian studies were graded at the Centre for Eye Research Australia, University of Melbourne using a computer-assisted vessel measurement system (Retinal Analysis; Optimate, Madison, WI, USA) that measured all vessels larger than $45 \mu \mathrm{m}$ in diameter [21, 27]. Trained graders masked to participant characteristics performed retinal vascular calibre measurements following a standard protocol in both centres. Briefly, for each image, all arterioles and venules coursing through an area of $0.5-1.0$ disc diameter from the optic disc margin were measured and summarised as central retinal arteriolar equivalent (CRAE) and central retinal venular retinal equivalent (CRVE) using the Parr-Hubbard formula [28] in ARIC and BDES, and Knudtson's revised formula [29] in MESA. These equivalents represent the average retinal vessel of calibres of the eye. In AusDiab and BMES, estimates obtained from the ParrHubbard formula using the Howard program were converted into estimates equivalent to IVAN measures using a newer formula termed the Parr-Hubbard-Knudtson formula [30]. Reproducibility of retinal vessel measurements were high for both CRAE and CRVE with intragrader and intergrader correlation coefficients ranging from 0.78 to 0.99 [28, 31].

Ascertainment of diabetes We defined incident diabetes as first occurrence at any follow-up examination. Diabetes definitions used by the individual studies were based on plasma or serum glucose levels and defined as fasting glucose level $\geq 7.0 \mathrm{mmol} / \mathrm{l}$, or $2 \mathrm{~h}$ glucose or casual glucose $\geq 11.1 \mathrm{mmol} / 1$, or $\mathrm{HbA}_{1 \mathrm{c}}>6.5 \%(47.5 \mathrm{mmol} / \mathrm{mol})$ or the use of diabetic medications with a previous physician's diagnosis. In ARIC, as retinal photographs were taken at visit three, this visit was considered as baseline for ascertainment of incident diabetes. After excluding individuals with prevalent diabetes at visit three, incident diabetes was defined as individuals with diabetes at the next visit after 3 years (visit four) or during the subsequent annual follow-up telephone calls from 4 to 
16 years. Similarly, in MESA, as retinal photographs were taken at visit two, this visit was considered as the baseline for ascertaining incident diabetes at visits three and four.

Assessment of covariates Information on covariates was collected from a combination of standardised interview and examination procedures in all studies. Data on demographic factors (age, sex, race/ethnicity), lifestyle (smoking, alcohol consumption), personal history of diseases and medication were collected using standardised questionnaires, and information on height, weight and BP measurements were from clinic examinations in each study. In all studies, a mean of two BP measurements was used as the BP value for the participants except for BMES in which BP was defined based on a single measurement. Ethnicity was categorised into white participants, black participants, and Mexican American/Asian/other participants. All studies included middle-aged and elderly participants except AusDiab, which included a wider age range (25-87 years).

Statistical analysis Both CRAE and CRVE were normally distributed and the pooled within-study SD was approximately $20 \mu \mathrm{m}$ for both arteriolar and venular calibres. We estimated the HR associated with each $20 \mu \mathrm{m}$ decrease in retinal arteriolar and each $20 \mu \mathrm{m}$ increase in retinal venular calibre in three models. Model 1 adjusted for age, sex and race/ethnicity (white participants, black participants, Mexican American/ Asian/other participants). Model 2 included variables from model 1 plus CVD factors including current smoking status (no, yes), current drinking status (no, yes), BMI $\left(\mathrm{kg} / \mathrm{m}^{2}\right.$ ), systolic BP (mmHg), hypertension (no, yes) and serum total cholesterol level (mmol/l). Model 3 included variables from model 2 plus baseline fasting glucose levels ( $\mathrm{mmol} / \mathrm{l})$. We estimated these separately for each study using a discrete time proportional hazards models. We pooled the log HR estimates of the different studies by random effects meta-analysis and displayed them in forest plots [32]. The extent of heterogeneity between studies was evaluated with the inconsistency $I^{2}$ statistic [33]. Validity of the above two-stage analyses results (with estimates of association calculated separately within each study, followed by pooling of the association estimates from different studies) was assessed by fitting a one-stage multilevel discrete time (Cox regression) random effect model using pooled individual data of all studies, with a random intercept to account for the study-specific effect. This onestage approach was also used to investigate exposurecovariate interactions because this approach provides a flexible way of examining individual-level interactions [34]. We performed subgroup analyses after interaction tests to detect evidence of any differences in hazards across levels of prespecified characteristics including age, sex, ethnicity, current smoking, BMI and hypertension status.
To test the robustness of our results, we performed several sensitivity analyses. First, we used an approach of leaving out one study at a time, calculating the pooled effect of the remaining studies and comparing the results with the combined effect based on all the studies. Second, to exclude the possibility that undetected diabetes or impaired fasting glucose had caused retinal microvascular changes, we repeated the analysis after excluding participants with follow-up of 5 years or less ( $n=5,587$ excluded). Third, we repeated the analysis after excluding participants with impaired fasting glucose at baseline $(n=4,683$ excluded), defined as a fasting plasma glucose level of $5.6 \mathrm{mmol} / \mathrm{l}$ to $<7.0 \mathrm{mmol} / \mathrm{l}$. Fourth, we repeated the analysis excluding individuals with incident diabetes defined exclusively by self-report (diabetes detected by annual telephone follow-up in ARIC; $n=1,439$ excluded). All analyses were performed using SAS version 9.3 (SAS Institute, Cary, NC, USA) and Review Manager 5 (The Nordic Cochrane Centre, The Cochrane Collaboration, Copenhagen, Denmark).

\section{Results}

Characteristics of the included study populations Table 1 shows the baseline characteristics of the 18,771 participants without diabetes from each of the five studies. Selected studies were published between 2002 (ARIC) and 2011 (MESA). The study sample sizes ranged from 768 in AusDiab to 7,899 in ARIC, and the majority of the participants were white (77.9\%). The number of follow-up visits ranged from one in AusDiab to four in BDES, and the median follow-up duration ranged from 3.1 years in MESA to 15.7 years in ARIC (including annual telephone follow-up). The cumulative incidence of diabetes ranged from $4.9 \%$ in MESA to $21.8 \%$ in ARIC. The proportion of participants older than 60 years ranged from $37.6 \%$ in AusDiab to $66.7 \%$ in BMES. BMES participants had the highest prevalence of hypertension (68.8\%) but the lowest prevalence of obesity (20.9\%). The mean CRAE ranged from $144.0 \mu \mathrm{m}$ in MESA participants to $193.9 \mu \mathrm{m}$ in BMES participants. The mean CRVE ranged from $192.3 \mu \mathrm{m}$ in ARIC participants to $230.2 \mu \mathrm{m}$ in BDES participants. Incident diabetes developed in 2,581 of the 18,771 participants (13.7\%).

Association of retinal vessel calibre with incidence of diabetes Figure 2a shows the study-specific and pooled HR of diabetes associated with a $20 \mu \mathrm{m}$ decrease in CRAE in the two-stage approach analyses. We considered HRs from the fully (model 3) adjusted model. The study-specific HRs for CRAE were not significant in most of the studies (ARIC, AusDiab, BDES and BMES) except in MESA. The pooled HR for CRAE was not significant (HR 0.95 [95\% CI 0.86, $1.06])$. 
Table 1 Baseline characteristics of participants in the five cohort studies $(n=18,771)$

\begin{tabular}{|c|c|c|c|c|c|}
\hline Characteristics & ARIC [19] & AusDiab [21] & BDES [20] & BMES [22] & MESA [17] \\
\hline Number of baseline population & 7,899 & 768 & 3,135 & 2,014 & 4,955 \\
\hline Cumulative incidence of diabetes, $\%$ (number of cases) & $21.8(1,723)$ & $10.8(83)$ & $12.1(379)$ & $7.6(153)$ & $4.9(243)$ \\
\hline $\begin{array}{l}\text { Follow-up durations after retinal measurement, median } \\
\text { (interquartile interval), years }\end{array}$ & $15.7(11.7-16.1)$ & $5.0(4.9-5.0)$ & $15.0(5.0-20.0)$ & $10.0(5.0-10.0)$ & $3.1(3.0-3.3)$ \\
\hline Year of baseline data collection & $1993-1995$ & 1999-2000 & $1988-1990$ & 1992-1994 & $2002-2004$ \\
\hline Year of most recent follow-up & 2012 & 2004-2005 & 2008-2010 & $2002-2004$ & 2006-2008 \\
\hline No. of follow-up visits & $14^{\mathrm{a}}$ & 1 & 4 & 2 & 2 \\
\hline Age at baseline, mean (SD), years & $59.4(5.6)$ & $55.4(12.6)$ & $59.7(10.4)$ & $63.8(8.4)$ & $62.7(10.0)$ \\
\hline Age range, years & $49-73$ & $25-87$ & $43-86$ & $49-93$ & $46-87$ \\
\hline \multicolumn{6}{|l|}{ Age group, years, $n(\%)$} \\
\hline$<60$ & $4,601(58.2)$ & $479(62.4)$ & $1,616(51.5)$ & $670(33.3)$ & $2,080(42.0)$ \\
\hline $60-69$ & $3,027(38.3)$ & $176(22.9)$ & $890(28.4)$ & $830(41.2)$ & $1,508(30.4)$ \\
\hline$\geq 70$ & $271(3.4)$ & $113(14.7)$ & $629(20.1)$ & $514(25.5)$ & $1,367(27.6)$ \\
\hline Men, $n(\%)$ & $3,423(43.3)$ & $331(43.1)$ & $1,369(43.7)$ & $833(41.4)$ & $2,333(47.1)$ \\
\hline \multicolumn{6}{|l|}{ Ethnicity, $n(\%)$} \\
\hline White & $6,575(83.2)$ & $768(100)$ & $3,135(100)$ & $2,014(100)$ & $2,126(42.9)$ \\
\hline Black & $1,324(16.8)$ & 0 & 0 & 0 & $1,248(25.2)$ \\
\hline Asian/Mexican Americans/other & 0 & 0 & 0 & 0 & $1,581(31.9)$ \\
\hline Current smoker, $n(\%)$ & $1,297(16.4)$ & $70(9.3)$ & $621(19.8)$ & $231(11.8)$ & $584(11.8)$ \\
\hline Current drinker, $n(\%)$ & $4,518(57.2)$ & $630(82.4)$ & $2,771(88.4)$ & $1,490(76.3)$ & $2,694(54.4)$ \\
\hline BMI, mean (SD), $\mathrm{kg} / \mathrm{m}^{2}$ & $27.8(5.1)$ & $27.8(5.3)$ & $28.6(5.3)$ & $26.2(4.3)$ & $28.0(5.2)$ \\
\hline \multicolumn{6}{|l|}{ BMI category, $n(\%), \mathrm{kg} / \mathrm{m}^{2}$} \\
\hline$<25.0$ & $2,442(30.9)$ & $246(32.2)$ & $773(24.7)$ & $855(42.8)$ & $1,503(30.3)$ \\
\hline 25.0 to 29.0 & $2,721(34.5)$ & $251(32.9)$ & $1,071(34.3)$ & $724(36.3)$ & $1,667(33.7)$ \\
\hline$\geq 30.0$ & $2,731(34.6)$ & $267(34.9)$ & $1,282(41.0)$ & $417(20.9)$ & $1,783(36.0)$ \\
\hline Systolic BP, mean (SD), mmHg & $122.2(17.9)$ & $133.7(18.5)$ & $129.9(19.1)$ & $144.2(20.1)$ & $122.8(20.2)$ \\
\hline Diastolic BP, mean (SD), mmHg & $71.6(10.2)$ & $71.1(11.7)$ & $77.9(10.4)$ & $83.4(9.5)$ & $70.5(10.0)$ \\
\hline Hypertension, $n(\%)$ & $2,691(34.1)$ & $345(45.2)$ & $1,427(45.5)$ & $1,384(68.8)$ & $2,216(44.7)$ \\
\hline Plasma glucose level, mean (SD), mmol/1 & $5.5(0.5)$ & $5.6(0.6)$ & $5.0(0.4)^{\mathrm{b}}$ & $4.9(0.5)$ & $5.1(0.5)$ \\
\hline Serum total cholesterol, mean (SD), mmol/1 & $5.4(1.0)$ & $5.6(1.0)$ & $6.0(1.1)$ & $6.1(1.1)$ & $4.9(0.9)$ \\
\hline CRAE, mean (SD), $\mu \mathrm{m}$ & $162.2(16.7)$ & $176.0(23.6)$ & $150.0(14.8)$ & $193.9(20.4)$ & $144.0(14.1)$ \\
\hline CRVE, mean (SD), $\mu \mathrm{m}$ & $192.3(16.3)$ & $206.2(22.2)$ & $230.2(22.4)$ & $225.4(19.8)$ & $213.3(21.5)$ \\
\hline
\end{tabular}

${ }^{\text {a }}$ In ARIC, one follow-up visit and 13 annual telephone follow-ups

${ }^{\mathrm{b}}$ Average blood glucose level in mmol/l converted from $\mathrm{HbA}_{1 \mathrm{c}}(\%)$

Figure $2 \mathrm{~b}$ shows the study-specific and pooled HR of diabetes associated with a $20 \mu \mathrm{m}$ increase in CRVE in the fully adjusted model. The HRs for CRVE were significant in two studies (AusDiab and BDES). However, the direction of association of CRVE with diabetes incidence was consistent with all HR $>1$ in the other studies. The pooled HR for CRVE was significant (HR 1.09 [95\% CI 1.02, 1.15]).

Table 2 shows the pooled hazard ratios of diabetes associated with CRAE and CRVE using the two-stage approach. In this model, CRAE was marginally associated with diabetes after adjusting for age, sex and race/ethnicity (in model 1). However, the association was not significant with additional adjustment for other traditional risk factors. CRVE was significantly associated with diabetes in model 1 . Although this association was attenuated with additional adjustment for traditional diabetes risk factors and fasting glucose levels in models 2 and 3, it remained significant in both models. Pooled estimates for CRAE (HR 0.95 [95\% CI 0.86, 1.06]) and CRVE $(1.09[1.02,1.15])$ in the fully adjusted models using the two-stage approach were similar to the estimates from the one-stage approach: CRAE (HR 0.98 [95\% CI 0.93, 1.03]) and CRVE $(1.08[1.03,1.12])$.

Tests for heterogeneity In analyses using the two-stage approach, the $p$ value for the heterogeneity test was 0.22 for CRVE, suggesting that there was no evidence of heterogeneity among the studies. By contrast, there was significant evidence 
a

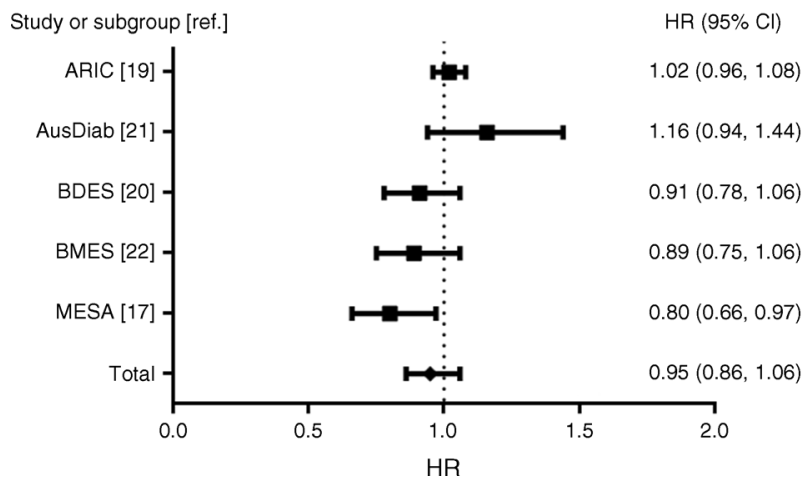

Fig. 2 (a) Forest plot of the adjusted HRs for incident diabetes by CRAE. Heterogeneity: $\tau^{2}=0.01 ; \chi^{2}=10.28, d f=4(p=0.04) ; I^{2}=61 \%$. Test for overall effect: $Z=0.96(p=0.34)$. Forest plot shows multivariable adjusted HRs (model 3) of diabetes per $20 \mu \mathrm{m}$ decrease in CRAE. (b) Forest plot of the adjusted hazard ratios for incident diabetes by CRVE. Heterogeneity: $\tau^{2}=0.00 ; \chi^{2}=5.76, d f=4(p=0.22) ; I^{2}=31 \%$. Test for overall effect: $Z=2.63(p=0.009)$. Forest plot shows multivariable adjusted HRs (model

for heterogeneity in the associations of CRAE with diabetes $(p=0.04)$.

Supplementary analyses In subgroup analyses stratified by selected participant characteristics (Table 3), the association of CRVE with diabetes was stronger in men than women $(p$ interaction $=0.03$ ) but remained consistent across categories of age, race/ethnicity, current smoking, BMI and hypertension ( $p$ interaction $>0.05$ ). In sensitivity analyses, recalculating the pooled risk estimates by leaving out one study at a time resulted in little change in the observed risk estimates for CRVE (HR 1.06 to 1.12). Second, when we repeated the analysis after excluding participants with less than 5 years of followup ( $n=12,870$ included), the association of CRVE with diabetes remained significant but slightly attenuated (HR $1.07[95 \%$ CI 1.01, 1.12]). Third, excluding participants with impaired fasting glucose at baseline ( $n=4,683$ excluded), the results were essentially similar $(1.10[1.03,1.17])$ for CRVE. In addition, excluding individuals with impaired fasting glucose at baseline and those with follow-up of less than 5 years $(n=9$, 327 excluded) did not alter the effect estimates (1.10 [1.02, b

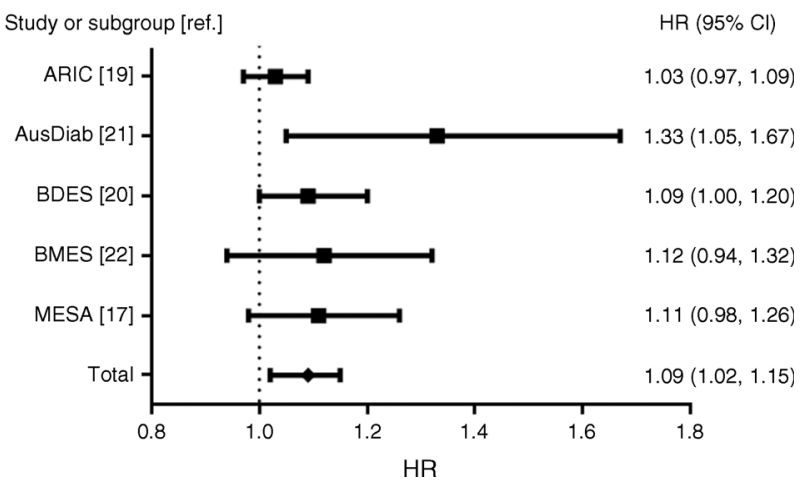

3) of diabetes per $20 \mu \mathrm{m}$ increase in CRVE. (a, b) The summary estimates presented were calculated using a random effects model. Squares indicate the individual HR in each study. The size of each square is proportional to the percentage weight of that individual study in the meta-analysis and the horizontal line indicates $95 \%$ CI. Pooled HR and $95 \% \mathrm{CI}$ are indicated by a black diamond

1.18]) for CRVE. Finally, excluding individuals with diabetes status defined exclusively by self-report (1,439 ARIC participants with diabetes identified during annual telephone follow-up were excluded) did not appreciably change the effect estimates for CRVE $(1.11[1.05,1.18])$.

\section{Discussion}

In this individual-level meta-analysis of 18,771 participants from five prospective cohort studies of individuals without diabetes at baseline, we showed that wider retinal venular calibre was associated with an increased risk of diabetes over a median follow-up of 10 years, independent of potential confounding factors. This association was stronger in men but was consistently present across categories of age, race/ethnicity, current smoking, BMI and hypertension status. The association of wider retinal venules with diabetes remained significant after excluding diabetes cases in the first 5 years of follow-up. Additionally, the relationship was evident after excluding individuals with impaired fasting glucose

Table 2 Overall HR of incident diabetes by retinal microvascular calibre, adjusted for risk factors

\begin{tabular}{|c|c|c|c|c|c|c|}
\hline \multirow[t]{2}{*}{ Progressive adjustment } & \multirow[t]{2}{*}{ No. of participants } & \multirow[t]{2}{*}{ No. of events } & \multicolumn{2}{|l|}{ CRAE } & \multicolumn{2}{|l|}{ CRVE } \\
\hline & & & Overall HR $(95 \% \mathrm{CI})$ & $I^{2}(p$ value $)$ & Overall HR $(95 \% \mathrm{CI})$ & $I^{2}(p$ value $)$ \\
\hline Model $1^{\mathrm{a}}$ & 18,741 & 2,577 & $1.04(0.99,1.09)$ & $68 \%(0.01)$ & $1.16(1.10,1.23)$ & $29 \%(0.23)$ \\
\hline Model $2^{\mathrm{b}}$ & 18,412 & 2,551 & $0.97(0.92,1.02)$ & $67 \%(0.02)$ & $1.10(1.05,1.15)$ & $0 \%(0.52)$ \\
\hline Model $3^{\mathrm{c}}$ & 18,198 & 2,540 & $0.95(0.86,1.06)$ & $61 \%(0.04)$ & $1.09(1.02,1.15)$ & $31 \%(0.22)$ \\
\hline
\end{tabular}

${ }^{\text {a }}$ Adjusted for age, sex, race/ethnicity (white, black, Mexican American/Asian/other)

${ }^{\mathrm{b}}$ Adjusted for variables in model $1+$ current smoking (no, yes), current drinking (no, yes), BMI ( $\mathrm{kg} / \mathrm{m}^{2}$ ), systolic blood pressure (mmHg), hypertension (no, yes), total cholesterol (mmol/l)

${ }^{c}$ Adjusted for variables in model 2+fasting glucose levels (mmol/l) 
Table 3 Overall HR of incident diabetes, by retinal microvascular calibre in subgroups

\begin{tabular}{|c|c|c|c|c|c|c|}
\hline \multirow[t]{2}{*}{ Variable } & \multirow[t]{2}{*}{ Persons at risk } & \multirow{2}{*}{$\begin{array}{l}\text { Incident cases } \\
\text { of diabetes }\end{array}$} & \multicolumn{4}{|l|}{$\mathrm{HR}(95 \% \mathrm{CI})^{\mathrm{a}}$} \\
\hline & & & $\mathrm{CRAE}^{\mathrm{b}}$ & $I^{2}(p$ value $)$ & $\mathrm{CRVE}^{\mathrm{c}}$ & $I^{2}(p$ value $)$ \\
\hline \multicolumn{7}{|l|}{ Age group, years } \\
\hline$<60$ & 9,446 & 1,491 & $0.99(0.92,1.06)$ & $3 \%(0.39)$ & $1.08(0.99,1.17)$ & $25 \%(0.25)$ \\
\hline $60-69$ & 6,431 & 877 & $0.96(0.86,1.07)$ & $13 \%(0.33)$ & $1.07(0.98,1.16)$ & $5 \%(0.38)$ \\
\hline$\geq 70$ & 2,892 & 213 & $0.99(0.78,1.26)$ & $50 \%(0.09)$ & $1.13(0.96,1.34)$ & $23 \%(0.27)$ \\
\hline \multicolumn{7}{|l|}{ Sex } \\
\hline Men & 8,289 & 1,161 & $0.95(0.81,1.11)$ & $62 \%(0.03)$ & $1.16(1.02,1.31)$ & $63 \%(0.03)$ \\
\hline Women & 10,482 & 1,420 & $0.95(0.86,1.05)$ & $30 \%(0.22)$ & $1.05(0.99,1.11)$ & $0 \%(0.61)$ \\
\hline \multicolumn{7}{|l|}{ Race/ethnicity } \\
\hline White & 14,618 & 2,026 & $0.97(0.88,1.06)$ & $40 \%(0.15)$ & $1.09(1.00,1.18)$ & $47 \%(0.11)$ \\
\hline Non-white & 4,153 & 555 & $0.94(0.76,1.15)$ & $60 \%(0.11)$ & $1.11(1.01,1.22)$ & $0 \%(0.72)$ \\
\hline \multicolumn{7}{|l|}{ Current smoker } \\
\hline Yes & 2,803 & 413 & $0.94(0.72,1.22)$ & $54 \%(0.07)$ & $1.01(0.91,1.13)$ & $0 \%(0.55)$ \\
\hline No & 15,866 & 2,160 & $0.96(0.86,1.06)$ & $54 \%(0.07)$ & $1.08(1.03,1.13)$ & $0 \%(0.45)$ \\
\hline \multicolumn{7}{|l|}{ BMI, $\mathrm{kg} / \mathrm{m}^{2}$} \\
\hline$<25.0$ & 5,857 & 393 & $0.93(0.82,1.06)$ & $0 \%(0.47)$ & $1.21(1.08,1.36)$ & $0 \%(0.50)$ \\
\hline$\geq 25.0$ & 12,914 & 2,188 & $0.97(0.88,1.06)$ & $43 \%(0.13)$ & $1.05(1.00,1.11)$ & $10 \%(0.35)$ \\
\hline \multicolumn{7}{|l|}{ Hypertension } \\
\hline Yes & 8,063 & 1,315 & $0.96(0.86,1.07)$ & $44 \%(0.13)$ & $1.09(1.01,1.17)$ & $25 \%(0.26)$ \\
\hline No & 10,663 & 1,254 & $0.92(0.81,1.06)$ & $44 \%(0.13)$ & $1.09(0.99,1.21)$ & $34 \%(0.20)$ \\
\hline
\end{tabular}

${ }^{a}$ Hazard ratios from discrete time proportional hazards model with retinal arteriolar and venular calibre as the main exposures, and age, sex, race/ethnicity, current smoker, current drinker, BMI, systolic BP, hypertension, total cholesterol level and fasting glucose level at baseline as covariates

${ }^{\mathrm{b}}$ Per $20 \mu \mathrm{m}$ decrease in CRAE

${ }^{\mathrm{c}}$ Per $20 \mu \mathrm{m}$ increase in CRVE

at baseline. Together, these findings suggest that wider retinal venular calibre is associated with an increase in the risk of diabetes. Retinal arteriolar calibre was not associated with diabetes.

Our study involving individual-level data $(n=18,771)$ is larger than the recent meta-analysis of this topic by Muris et al, which used published estimates from prospective cohort studies [24]. The larger sample size of the current study provided sufficient power to perform detailed analyses and to investigate the possibility of reverse causality. Previous studies have not used consistent methods to report these associations. Discrepancy in the results of the meta-analysis by Muris et al and our meta-analysis could be due to differences in the inclusion of studies. For example, some studies used the retinal AVR for analysis (ARIC, BDES and the Rotterdam Study) while others (AusDiab, BDES, BMES and the Rotterdam Study) included separate analysis for retinal arteriolar and venular calibre. Muris et al used four studies (AusDiab, BDES, BMES and the Rotterdam Study; $n=8,487$ ) to examine the individual association of retinal arteriolar and venular diameter with diabetes, and three studies (ARIC, BDES and the Rotterdam study; $n=13,554$ ) to examine the association of retinal AVR with diabetes [24].
In the meta-analysis by Muris et al, while smaller AVR was significantly associated with diabetes (RR 1.18 [95\% CI 1.08, $1.29])$, neither smaller arteriole $(1.08[0.96,1.22])$ nor wider venule $(1.06[0.97,1.15])$ alone was associated with diabetes. The lack of association of retinal arteriole or venule with diabetes could be due to a smaller sample size or shorter followup; for example, the published ARIC study was restricted to participants with data on incident diabetes of up to 3 years follow-up, whereas in the current study we included incident data up to 16 years of follow-up. In addition, aggregate-level meta-analyses based on published estimates from different studies do not account for differences in analytical methods and covariate adjustment. For example, some studies adjusted for systolic BP [21, 22, 35], while others adjusted for both systolic and diastolic BP [20] or mean arterial BP [19]; some studies used Cox proportional hazards model to estimate relative risks or HRs [20,35], whereas others used logistic regression models to estimate odds ratios [19, 21, 22].

Furthermore, in the meta-analysis by Muris et al, retinal variables were only one of several microvascular variables assessed (including plasma markers of endothelial dysfunction, peripheral vascular reactivity, retinal vascular calibre and microalbuminuria); therefore, no subgroup analyses or 
sensitivity analyses were performed. In the current individuallevel meta-analysis, we have performed detailed subgroup and sensitivity analyses besides standardising the analytical methods and covariate adjustment. Furthermore, the studies included in the current meta-analysis displayed good quality by the prospective design, comparable assessment of retinal vascular variables, objective assessment of outcome and information on covariates. Thus, we believe our estimates are robust and less biased than those of Muris et al.

\section{Mechanism of association between wider retinal venules} and diabetes The exact pathogenesis underlying the association of wider retinal venules with diabetes is not clear. Evidence from experimental studies suggests that microvascular dysfunction appears early in the course of insulin resistance, leading to impaired glucose delivery and glucose uptake $[5,36]$. In rats, insulin has been shown to be involved in capillary recruitment in skeletal muscle, which is mediated by a nitric-oxide-dependent pathway [6] or by TNF- $\alpha$ (a marker of inflammation), leading to impaired insulin-mediated capillary recruitment and glucose uptake [37]. This evidence supports the role of microvascular dysfunction in altered glucose metabolism. Association of wider retinal venules with markers of inflammation and endothelial dysfunction (such as C-reactive protein, IL-6, fibrinogen, leucocyte count, soluble intracellular adhesion molecule and plasminogen activator inhibitor) have also been reported [11-14]. This evidence suggests that retinal venules widen in response to inflammation; thus, endothelial dysfunction may represent impaired microvascular reactivity, insulin resistance and impaired glucose metabolism [38].

Strengths and limitations Our study has several strengths. First, analysis of data from individual participants rather than published estimates has allowed us to pool data by standardising the inclusion criteria of study participants and to include adjustment for potential risk factors. Second, retinal vascular measurement using digital retinal photography is a fairly new technique; therefore, we were able to collaborate with most of the researchers worldwide who have used this technology in prospective studies to assess the risk of diabetes. In addition, as there were no studies published in any language other than English that met the study criteria, we feel that publication bias is highly unlikely. Unpublished studies, if any, are likely to be few since the results of large prospective studies (even if findings are negative) are more likely to be reported or known to the research community. Thus, we believe the impact of unpublished results on the pooled estimate reported herein (based on 2,581 incident cases of diabetes) would be negligible. Third, inclusion of only prospective studies and the persistence of association of wider retinal venule with diabetes after exclusion of the first 5 years of follow-up eliminate the possibility of reverse causality explaining the observed association.

Our study has some limitations. First, we cannot exclude the possibility of residual confounding due to unmeasured or unknown risk factors. For example, we did not have information on the genetic profiles of participants in all studies. However, previous genome-wide association studies on retinal venular diameter, using data from some of the cohorts in our metaanalysis, do not reveal genetic markers common to both diabetes $[39,40]$ and retinal venular diameter [30]; thus, significant confounding from such interactions are unlikely to have influenced the association reported here. In addition, although we excluded individuals with prevalent CVD, the presence of subclinical CVD or other causes of inflammation may have affected our results. We believe, however, that the influence from residual confounding is likely to be minimal, as accounting for a large number of risk factors using information available from all cohorts did not materially alter the results.

Second, ascertainment of diabetes was based on casual plasma glucose and self-report history of diabetes in BDES and based on self-reported history alone in the annual followup data in ARIC. Thus, we cannot exclude some misclassification of outcome. However, results were similar in studies using fasting plasma glucose to define diabetes (e.g. BMES). A previous validation study conducted using a subsample of 1,738 ARIC participants in 2004-2005 reported self-reported diabetes to be $>90 \%$ specific and reliable compared with diabetes defined using a combination of glucose or $\mathrm{HbA}_{1 \mathrm{c}}$ measurements or medication use [41].

Third, CRAE and CRVE measurements were different across the studies possibly due to differences in the software versions employed. These differences could have resulted in measurement error. For example, CRAE measurements were higher in BMES $(193.9 \mu \mathrm{m})$ while CRVE measurements were higher in BDES $(230.2 \mu \mathrm{m})$ than in other studies. However, the finding of a similar direction of association of CRVE with diabetes in all studies, lack of evidence of heterogeneity $(p=$ 0.22 ) among the studies and similar estimates of association in the 'leaving one study out approach' in sensitivity analyses suggest that our estimates are robust despite the difference in measurements. Fourth, we did not include the Rotterdam Study in the meta-analysis, but the published results from the Rotterdam Study were very similar to those of the present study (OR 1.09 [95\% CI 0.90, 1.33]); therefore, we believe that omission of the Rotterdam Study did not affect our study findings. Finally, the reported association between wider CRVE and incident diabetes is low in magnitude (10\% increase in risk per $20 \mu \mathrm{m}$ increase in CRVE calibre), and its use in clinical practice for diabetes prediction is questionable.

Conclusions In summary, our meta-analysis of prospective studies of diverse populations shows that wider venular calibre in the retinal vessels is associated with an increased risk of 
type 2 diabetes. The consistency of associations across five different populations and across subgroups suggests that microvascular processes associated with wider retinal venular calibre may be a signal for the underlying pathogenesis leading to clinical diabetes. Further studies are warranted to test the applicability and clinical usefulness of retinal imaging in predicting the risk of diabetes.

Acknowledgements The authors thank the staff and participants of the ARIC study, the AusDiab study, BDES, BMES and MESA for their valuable contributions. The authors also thank R.B.B. Mohamed Abdul (Singapore Eye Research Institute, Singapore, Republic of Singapore) for her help with the figures and formatting the manuscript.

Duality of interest The authors declare that there is no duality of interest associated with this manuscript.

Contribution statement All authors contributed to the intellectual development of this paper. TYW and CS designed the study. TYW acquired data from the collaborators: MFC, PM, RK and ES. CS supervised data analysis and wrote the initial draft. WKL performed the statistical analyses. BEKK, CS, WKL, RK, MFC, JJW, PM, JES, ES, ARS and TYW assisted in interpretation of the analysed data and provided critical corrections of the manuscript. TYW is the guarantor of this work and as such had full access to all the data in the study and takes responsibility for the integrity of the data and accuracy of the data analysis. The final version of the paper has been seen and approved by all the authors.

Funding We used restricted access datasets of the ARIC study and MESA. The ARIC study is carried out as a collaborative study supported by National Heart, Lung, and Blood Institute contracts (HHSN268201100005C, HHSN268201100006C, HHSN268201100007C, HHSN268201100008C, HHSN268201100009C, HHSN268201100010C, HHSN268201100011C and HHSN268201100012C). MESA was supported by contracts N01-HC-95159 through N01-HC-95165 from the National Heart, Lung and Blood Institute and NIH Intramural Research award ZIAEY000403 from the National Eye Institute, USA. This article does not necessarily convey the opinions or views of the ARIC study, MESA or the National Heart, Lung and Blood Institute. The AusDiab study was supported by National Health and Medical Research Council Grants 350448 and 233200, and a Sylvia and Charles Viertel Charitable Foundation grant. BDES was supported by the National Eye Institute, National Institutes of Health Grant EY-06594, and by Research to Prevent Blindness, New York, NY, USA. The BMES was supported by the Australian National Health \& Medical Research Council. The study was funded by grants from the National Medical Research Council, Singapore (STaR/0003/2008 and CG/SERI/2010), which had no role in study design, data analysis or interpretation of results.

\section{References}

1. International Diabetes Federation (2013) IDF diabetes atlas update 2012. International Diabetes Federation, Brussels

2. Department of Health and Human Services, Centers for Disease Control and Prevention (2012) National diabetes fact sheet: national estimates and general information on diabetes and prediabetes in the United States, 2011. Centers for Disease Control and Prevention, Atlanta
3. Clark MG, Barrett EJ, Wallis MG, Vincent MA, Rattigan S (2002) The microvasculature in insulin resistance and type 2 diabetes. Semin Vasc Med 2:21-31

4. Jaap AJ, Shore AC, Tooke JE (1997) Relationship of insulin resistance to microvascular dysfunction in subjects with fasting hyperglycaemia. Diabetologia 40:238-243

5. Clark MG (2008) Impaired microvascular perfusion: a consequence of vascular dysfunction and a potential cause of insulin resistance in muscle. Am J Physiol Endocrinol Metab 295:E732-E750

6. Vincent MA, Barrett EJ, Lindner JR, Clark MG, Rattigan S (2003) Inhibiting NOS blocks microvascular recruitment and blunts muscle glucose uptake in response to insulin. Am J Physiol Endocrinol Metab 285:E123-E129

7. Struijker-Boudier HA, Heijnen BF, Liu YP, Staessen JA (2012) Phenotyping the microcirculation. Hypertension 60:523-527

8. Struijker-Boudier HA, Rosei AE, Bruneval P et al (2007) Evaluation of the microcirculation in hypertension and cardiovascular disease. Eur Heart J 28:2834-2840

9. Liew G, Wang JJ, Mitchell P, Wong TY (2008) Retinal vascular imaging: a new tool in microvascular disease research. Circ Cardiovasc Imaging 1:156-161

10. Kim JA, Montagnani M, Koh KK, Quon MJ (2006) Reciprocal relationships between insulin resistance and endothelial dysfunction: molecular and pathophysiological mechanisms. Circulation 113:1888-1904

11. Daien V, Carriere I, Kawasaki R et al (2013) Retinal vascular caliber is associated with cardiovascular biomarkers of oxidative stress and inflammation: the POLA study. PLoS One 8, e71089

12. Ikram MK, de Jong FJ, Vingerling JR et al (2004) Are retinal arteriolar or venular diameters associated with markers for cardiovascular disorders? The Rotterdam Study. Invest Ophthalmol Vis Sci 45:2129-2134

13. Klein R, Klein BE, Knudtson MD, Wong TY, Tsai MY (2006) Are inflammatory factors related to retinal vessel caliber? The Beaver Dam Eye Study. Arch Ophthalmol 124:87-94

14. Wong TY, Islam FM, Klein R et al (2006) Retinal vascular caliber, cardiovascular risk factors, and inflammation: the multi-ethnic study of atherosclerosis (MESA). Invest Ophthalmol Vis Sci 47:2341-2350

15. Jeganathan VS, Sabanayagam C, Tai ES et al (2009) Retinal vascular caliber and diabetes in a multiethnic Asian population. Microcirculation 16:534-543

16. Kifley A, Wang JJ, Cugati S, Wong TY, Mitchell P (2007) Retinal vascular caliber, diabetes, and retinopathy. Am J Ophthalmol 143:1024-1026

17. Nguyen TT, Wang JJ, Sharrett AR et al (2008) Relationship of retinal vascular caliber with diabetes and retinopathy: the Multi-Ethnic Study of Atherosclerosis (MESA). Diabetes Care 31:544-549

18. Cheung CY, Ikram MK, Klein R, Wong TY (2015) The clinical implications of recent studies on the structure and function of the retinal microvasculature in diabetes. Diabetologia 58:871-885

19. Wong TY, Klein R, Sharrett AR et al (2002) Retinal arteriolar narrowing and risk of diabetes mellitus in middle-aged persons. JAMA 287:2528-2533

20. Wong TY, Shankar A, Klein R, Klein BE, Hubbard LD (2005) Retinal arteriolar narrowing, hypertension, and subsequent risk of diabetes mellitus. Arch Intern Med 165:1060-1065

21. Nguyen TT, Wang JJ, Islam FM et al (2008) Retinal arteriolar narrowing predicts incidence of diabetes: the Australian Diabetes, Obesity and Lifestyle (AusDiab) Study. Diabetes 57:536-539

22. Kifley A, Wang JJ, Cugati S, Wong TY, Mitchell P (2008) Retinal vascular caliber and the long-term risk of diabetes and impaired fasting glucose: the Blue Mountains Eye Study. Microcirculation $15: 373-377$ 
23. Ikram MK, Janssen JA, Roos AM et al (2006) Retinal vessel diameters and risk of impaired fasting glucose or diabetes: the Rotterdam study. Diabetes 55:506-510

24. Muris DM, Houben AJ, Schram MT, Stehouwer CD (2012) Microvascular dysfunction is associated with a higher incidence of type 2 diabetes mellitus: a systematic review and meta-analysis. Arterioscler Thromb Vasc Biol 32:3082-3094

25. Stroup DF, Berlin JA, Morton SC et al (2000) Meta-analysis of observational studies in epidemiology: a proposal for reporting. Meta-analysis Of Observational Studies in Epidemiology (MOOSE) group. JAMA 283:2008-2012

26. Muntner P, Woodward M, Mann DM et al (2010) Comparison of the Framingham Heart Study hypertension model with blood pressure alone in the prediction of risk of hypertension: the Multi-Ethnic Study of Atherosclerosis. Hypertension 55:1339-1345

27. Tikellis G, Wang JJ, Tapp R et al (2007) The relationship of retinal vascular calibre to diabetes and retinopathy: the Australian Diabetes, Obesity and Lifestyle (AusDiab) study. Diabetologia 50:2263-2271

28. Hubbard LD, Brothers RJ, King WN et al (1999) Methods for evaluation of retinal microvascular abnormalities associated with hypertension/sclerosis in the Atherosclerosis Risk in Communities Study. Ophthalmology 106:2269-2280

29. Knudtson MD, Lee KE, Hubbard LD, Wong TY, Klein R, Klein BE (2003) Revised formulas for summarizing retinal vessel diameters. Curr Eye Res 27:143-149

30. Ikram MK, Sim X, Jensen RA et al (2010) Four novel Loci (19q13, $6 \mathrm{q} 24,12 \mathrm{q} 24$, and $5 \mathrm{q} 14)$ influence the microcirculation in vivo. PLoS Genet 6, e1001184

31. Wong TY, Knudtson MD, Klein R, Klein BE, Meuer SM, Hubbard LD (2004) Computer-assisted measurement of retinal vessel diameters in the Beaver Dam Eye Study: methodology, correlation between eyes, and effect of refractive errors. Ophthalmology 111:1183-1190
32. DerSimonian R, Laird N (1986) Meta-analysis in clinical trials. Control Clin Trials 7:177-188

33. Higgins JP, Thompson SG, Deeks JJ, Altman DG (2003) Measuring inconsistency in meta-analyses. BMJ 327:557-560

34. Thompson S, Kaptoge S, White I, Wood A, Perry P, Danesh J (2010) Statistical methods for the time-to-event analysis of individual participant data from multiple epidemiological studies. Int J Epidemiol 39:1345-1359

35. Yau JW, Xie J, Lamoureux E et al (2012) Retinal microvascular calibre and risk of incident diabetes: the multi-ethnic study of atherosclerosis. Diabetes Res Clin Pract 95:265-274

36. Clark MG, Wallis MG, Barrett EJ et al (2003) Blood flow and muscle metabolism: a focus on insulin action. Am J Physiol Endocrinol Metab 284:E241-E258

37. Youd JM, Rattigan S, Clark MG (2000) Acute impairment of insulin-mediated capillary recruitment and glucose uptake in rat skeletal muscle in vivo by TNF- $\alpha$. Diabetes 49:1904-1909

38. Balletshofer BM, Rittig K, Enderle MD et al (2000) Endothelial dysfunction is detectable in young normotensive first-degree relatives of subjects with type 2 diabetes in association with insulin resistance. Circulation 101:1780-1784

39. Cornes BK, Brody JA, Nikpoor N et al (2014) Association of levels of fasting glucose and insulin with rare variants at the chromosome 11p11.2-MADD locus: Cohorts for Heart and Aging Research in Genomic Epidemiology (CHARGE) Consortium Targeted Sequencing Study. Circ Cardiovasc Genet 7:374-382

40. Chen P, Takeuchi F, Lee JY et al (2014) Multiple nonglycemic genomic loci are newly associated with blood level of glycated hemoglobin in East Asians. Diabetes 63:2551-2562

41. Schneider AL, Pankow JS, Heiss G, Selvin E (2012) Validity and reliability of self-reported diabetes in the atherosclerosis risk in communities study. Am J Epidemiol 176:738-743 